\title{
Collaborative care improved the cost effectiveness of treatment for major depression in primary care
}

\author{
Von Korff M, Katon W,Bush T, et al. Treatment costs, cost offset, and cost-effectiveness of collaborative management of depression. \\ Psychosom Med 1998 Mar/Apr;60:143-9.
}

\section{Question}

What are the treatment costs, cost offset effects, and cost effectiveness of collaborative care of depression in primary care?

\section{Design}

Cost analysis using data from 2 randomised controlled trials each with 12 months follow up.

\section{Setting}

Group Health Cooperative of Puget Sound, Seattle, Washington, USA.

\section{Patients}

370 patients between 18 and 80 years of age who were starting antidepressant medication for definite or probable major depression as diagnosed by their primary care physician. Exclusion criteria were current alcohol abuse, psychotic symptoms, or dementia; pregnancy; or terminal illness. Follow up and cost data were available for 332 patients $(90 \%)$.

\section{Intervention}

Patients were allocated to collaborative care or usual care. Collaborative care consisted of psychiatrist delivered enhanced management of pharmacotherapy and brief psychoeducational interventions to enhance adherence (trial 1) or brief cognitive behavioural therapy and enhanced patient education delivered by a clinical psychologist (trial 2).

\section{Main cost and outcome measures}

Costs of treating depression (average direct and overhead costs), cost offset (mean costs among intervention patients minus mean costs among control patients), and cost effectiveness (average costs divided by treatment effectiveness). Costs are in US dollars.

\begin{abstract}
Main results
Collaborative care increased the costs of treating depression mainly because of the extra visits required to provide the interventions. The annual cost of collaborative care ranged from $\$ 1045$ to $\$ 1337$ compared with usual care cost of $\$ 900$ for patients with major depression and between $\$ 500$ and $\$ 700$ for patients with minor depression. In both trials, the costs of specialty mental health services were lower among patients with major depression who received collaborative care rather than usual care $(\$ 123 v \$ 317, \mathrm{p}=0.03)$. This cost offset in specialty mental health services was not seen among patients with minor depression. The costs of ambulatory medical care services did not differ between the treatment groups. Among patients with major depression there was a modest increase in cost effectiveness. In patients with major depression, the cost for each patient successfully treated (defined as a $\geqslant 50 \%$ reduction in the Hopkins Symptom Checklist-90 depression symptom score at 4 mo follow up) was lower for collaborative care than for usual care (\$1679 [trial 2] to $\$ 1797$ [trial 1] v \$1941 to $\$ 2170)$. For patients with minor depression, collaborative care was more costly and not more cost effective than usual care.
\end{abstract}

\section{Conclusions}

Collaborative care increased the costs of treating depression but improved the cost effectiveness of treatment for patients with major depression. A cost offset was seen for major depression in specialty mental health costs, but not in medical care costs.

Source of funding: National Institute of Mental Health.

For correspondence: Dr M Von Korff, Center for Health Studies, Group Health Cooperative of Puget Sound, 1730 Minor Avenue, Suite 1600, Seattle, WA 98101, USA. Fax 1+206 2872871.

\section{Commentary}

Depression has always been a prevalent, pernicious disease, but its morbidity, mortality, and costs are only now receiving recognition. As depression is largely treated in primary care settings, estimating treatment costs and cost effectiveness of providing collaborative care in these settings is essential.

This cost benefit analysis by Von Korff $e t$ al of 2 randomised controlled trials provides valuable evidence that, although increasing the costs, the effectiveness of the treatment of patients suffering from major depression can be improved with collaborative care. Similar findings were reported in another recent randomised primary care study. ${ }^{1}$ Although from a very specific context-a white and highly educated population-these data suggest that collaborative care is useful to achieve effective treatment with only a modest increment in the cost by targeting specific populations.
Enhancing services for patients with minor depression was not found to be more effective nor more cost effective than usual care. Lack of statistical power may be an explanation of these findings. A Cochrane systematic review suggests that patients suffering from dysthmia or depressive neurosis can improve with antidepressant medication. ${ }^{2}$ In this study by Von Korff et al, patients with minor depressive disorders improved equally as well under both collaborative care and usual care. Given the high prevalence of minor depression it is important to evaluate if such approaches may increase the value of treatment for minor depression. This would require larger randomised trials, which are now underway.

A further clinical thought: caution should be used when considering broad aspects of mental health policy-issues other than narrow economic evaluation need special attention (such as the cost of unsuccessful treatments). As Archie Cochrane said: "Surely priority should be given to finding out which treatments are effective and then ensuring that these treatments are efficiently given to all who need them.".

Mauricio Silva de Lima, MD, PhD Universidade Federal de Pelotas Pelotas, RS, Brazil

1 Katzelnick DJ, Simon GE, Pearson SD, et al. Randomized trial of a depression management program in high utilizers of medical care. Toronto: Annual Meeting of American Psychiatric Association, 1998

2 Lima MS, Moncrieff J. A comparison of drugs versus placebo for the treatment of dysthymia: a systematic review (Cochrane Review). In: Cochrane Library, issue 2, 1998. Oxford: Cochrane Library,

3 Cochrane AL. Effectiveness and efficiency: random reflections on health services. Second edition. London: Nuffield Provincial Hospitals Trust, 1989. 